\section{TROMBOEMBOLISMO VENOSO: PROFILAXIA EM PACIENTES CLÍNICOS - PARTE I}

Autoria: Academia Brasileira de Neurologia, Associação de Medicina Intensiva Brasileira, Federação Brasileira das Associações de Ginecologia e Obstetrícia, Sociedade Brasileira de Angiologia e de Cirurgia Vascular, Sociedade Brasileira de Cancerologia, Sociedade Brasileira de Cardiologia, Sociedade Brasileira de Clínica Médica, Sociedade Brasileira de Geriatria e Gerontologia, Sociedade Brasileira de Hematologia e Hemoterapia, Sociedade Brasileira de Pneumologia e Tisiologia, Sociedade Brasileira de Reumatologia

Participantes: Ana Thereza Rocha, Edison Ferreira de Paiva, Arnaldo Lichtenstein, Rodolfo Milani J unior, Cyrillo Cavalheiro Filho, Francisco Humberto de Abreu Maffei, Charles André, Marcia Maiumi Fukujima, Gabriel R. de Freitas, Silvia Lage, Claudio Bonduki, Carlos Carvalho, Eduardo Ramacciotti, Vera Lúcia Teixeira, Paulo Roberto Mattos da Silveira, Clarissa Mathias, José Carlos Nicolau, Renato Delascio Lopes, Salo Buksman, Verônica Hagemeyer Santos, Élbio D’Amico, Mário Terra-Filho, Roger Abramino Levy, Alexandre Wagner Silva de Souza

\section{Descrição do método de coleta de evidência:}

A pesquisa envolveu os seguintes bancos de dados: a) Medline (de 1966 a agosto de 2004), b) Base de Dados Cochrane de Revisões Sistemáticas (Cochrane Library), c) LILACS (de 1985 a 2004), d) resumos de estudos publicados em encontros científicos das sociedades envolvidas, e) referências cruzadas do material levantado. A estratégia utilizada no Medline foi ("Thrombosis"[MeSH] OR "Venous Thrombosis"[MeSH] OR "Embolism and Thrombosis"[MeSH] OR "Embolism"[MeSH] OR "Pulmonary Embolism"[MeSH]) NOT "Atrial Fibrillation"[MeSH] NOT ("Surgery"[MeSH] OR Surgery" [Subheading] OR "Surgical Procedures, Operative"[MeSH]) NOT ("Wounds and Injuries"[MeSH] OR "injuries"[Subheading] OR "Multiple Trauma"[MeSH])".

\section{Graus de recomendação e força de evidência:}

A: Estudos experimentais ou observacionais de melhor consistência.

B: Estudos experimentais ou observacionais de menor consistência.

C: Relatos de casos (estudos não controlados).

D: Opinião desprovida de avaliação crítica, baseada em consensos, estudos fisiológicos ou modelos animais.

\section{Introdução}

Tromboembolismo venoso (TEV) representa um espectro de doenças que inclui trombose venosa profunda, trombose associada a cateteres venosos centrais e, a complicação mais grave, tromboembolismo pulmonar (TEP). Tanto episódios assintomáticos quanto aqueles clinicamente aparentes em pacientes hospitalizados estão associados à fatalidade. Em hospitais brasileiros e de todo o mundo, a suspeita antemortem é feita em pequena proporção dos TEP. A ênfase dada à incidência de TEV em populações cirúrgicas despertou a necessidade de profilaxia para este grupo de pacientes. No entanto, mais da metade dos pacientes que sofrem TEV sintomático não têm afecções cirúrgicas, mas sim clínicas.

Os fatores de risco para TEV em pacientes não submetidos à cirurgia e as medidas profiláticas são abordados nas diretrizes de profilaxia de TEV em pacientes clínicos, dividida em três partes. Na primeira parte, encontram-se as informações sobre: mobilidade reduzida, idade $=55$ anos, história prévia de TEV, varizes, insuficiência venosa crônica, insuficiência arterial periférica, obesidade, trombofilias hereditárias e adquiridas. $\mathrm{N}$ a segunda parte: gravidez, pós-parto, reposição hormonal, contraceptivos, insuficiência cardíaca congestiva classes IIIIV, infarto agudo do miocárdio, acidente vascular cerebral isquêmico, acidente vascular cerebral hemorrágico, paresia e paralisia de membros inferiores. $\mathrm{Na}$ terceira parte: doença reumatológica ativa, doença inflamatória intestinal, doenças respiratórias graves, infecções com exceção das torácicas, síndrome nefrótica, câncer, quimioterapia, hormonioterapia, internação em unidade de terapia intensiva, uso de cateteres venosos centrais, uso de cateteres para hemodiálise, uso de Swan-Ganz. Versão integral e extensa que originou essas diretrizes encontra-se documento elaborado pelo Grupo de Estudos de Trombose e Hemostasia.

Para identificar os estudos, uma pesquisa auxiliada por computador foi feita independentemente por cada representante das sociedades e pelos coordenadores para cada um dos assuntos do parágrafo acima. Procurou-se, também, estudos avaliando a eficácia dos métodos de profilaxia contra TEV, nesta população, incluindo heparina não fracionada (HNF), heparinas de baixo peso molecular (HBPM) e métodos físicos.

A seleção dos estudos foi baseada na leitura do título e do resumo e, quando necessário, na leitura do texto completo por pelo menos dois investigadores de cada área. A avaliação da qualidade dos estudos baseou-se em roteiros padronizados, considerando-se: desenho do estudo, randomização, recrutamento de pacientes consecutivos, seguimento apropriado, método de detecção de TEV, avaliação mascarada, precisão dos resultados e aplicabilidade dos resultados às questões clínicas sobre fatores de risco ou eficácia da profilaxia contra TEV. Como se avaliou, nesta revisão, tanto o risco quanto a eficácia de profilaxia contra TEV como desfechos principais dos estudos, foram incluídos, preferencialmente, ensaios clínicos controlados e randomizados (ECCR), mas também estudos de coorte e caso-controle.

Foram coletados: desenho do estudo, número de pacientes recrutados e analisados, características da população investigada, tipo de teste de triagem usado para detecção de TEV e incidência de TEV no grupo com o fator de risco, com e sem uso de profilaxia, se aplicável. Quando possível, foram também coletados ou calculados o risco relativo $(R R)$ ou a razão de chances ("odds ratio" ou OR), a redução do risco relativo (RRR) e o número necessário para causar danos ("number needed to harm" ou NNH), para estudos investigando risco, e o número necessário tratar ("number needed to treat" ou NNT), para estudos investigando benefícios da profilaxia, e o intervalo de confiança (IC). 


\section{Mobilidade reduzida}

A avaliação de 1.000 pacientes submetidos a ultra-sonografia por suspeita de TEV mostrou correlação significativa entre perda da mobilidade há mais de 3 dias e a probabilidade de desenvolver trombose venosa profunda $(\text { TVP })^{1}(\mathbf{B})$. Em estudo epidemiológico caso-controle com 1.272 pacientes ambulatoriais $^{2}(\mathbf{B})$, tanto a permanência em pé por mais de 6 horas por dia quanto a imobilização representada por confinamento ao leito ou poltrona foram associados a risco aumentado de TEV (OR 1,9; IC de 95\% 1,1-3,1 e OR 5,6; IC de 95\% 2,3-13,7, respectivamente). Do mesmo modo, outro estudo ${ }^{3}$ (B) mostrou que confinamento em hospital ou casa de repouso aumentava 0 risco de TEV (OR 8,0; IC de 95\% 4,5-14,2). Perdas de mobilidade mais acentuadas, como incapacidade para caminhar independentemente mais do que 10 metros, foram mais frequentemente relacionadas ao aparecimento de $\operatorname{TEV}{ }^{4}(\mathbf{C})$. Em estudo caso-controle recente, realizado em pacientes internados e com idade acima de 65 anos, identificou-se a presença de mobilidade reduzida como fator de risco independente para o desenvolvimento de TEV, com OR variando de 1,73 até 5,64, dependendo do grau de mobilidade $(\mathbf{B})$.

\section{Idade}

Vários estudos epidemiológicos têm mostrado que a incidência de TEV aumenta exponencialmente com a idade, não sendo claro se por alterações dos mecanismos de coagulação ou pela presença de comorbidades trombogênicas. Em estudo realizado em Oslo, a incidência de TEV aumentou de 1:10.000 aos 20 anos de idade, para 1:1.000 aos 50 anos ${ }^{6}$ (C). No estudo norte-americano em Worcester, mostrou-se que a partir da 5 a década de vida, o risco de TEV praticamente dobrou a cada década subseqüente (RR 1,9) e que a incidência de TEV foi superior à cifra de 62:100.000, a partir dos 50 anos ${ }^{7}(\mathbf{B})$.

Estudo de coorte mostrou que a adição de meias elásticas à quimioprofilaxia, em pacientes com idade $>64$ anos, diminui ainda mais o risco de TEV quando comparado com quimioprofilaxia isolada ou com nenhuma profilaxia, $10,4 \%$, vs. $13,8 \%$ vs. $20,8 \%$, respectivamente ${ }^{8}(\mathbf{B})$.

\section{História prévia de TEV}

História pessoal prévia é fator de risco em vários cenários: pacientes internados, pacientes ambulatoriais e na população geral. Trombofilia persistente ou lesão residual do sistema venoso podem favorecer a recorrência da condição. 0 estudo caso-controle ${ }^{2}(\mathbf{B})$ revelou uma forte predisposição para novos eventos tromboembólicos em pacientes ambulatoriais com história pessoal de TEV (OR 15,6). Estudo ambulatorial ${ }^{9}$ (C) mostrou que pacientes clínicos com suspeita de TVP e história prévia de TEV tinham chance aumentada de confirmar 0 diagnóstico com flebografia (OR 1,68). Não há estudos cujo objetivo principal foi responder sobre a eficácia de profilaxia em pacientes clínicos agudamente enfermos, com história prévia de TEV.

\section{Insuficiência vascular periférica}

0 impacto de varizes dos membros inferiores como fator de risco adicional para TEV, em pacientes clínicos, é controverso.
Entre outros motivos, a descrição clínica da gravidade das varizes é bastante subjetiva e, portanto, variável entre os estudos. Além disto, trabalhos investigando este problema são escassos e não há relatos de que o risco potencial de TEV diminui com a remoção cirúrgica das veias varicosas. Em uma coorte de 547 pacientes clínicos com TEV, 47\% não apresentavam nenhuma causa predisponente para trombose, mas destes, 42\% (108/ 258) tinham varizes ${ }^{10}(\mathbf{C})$. Em estudo populacional do tipo casocontrole, em pacientes clínicos, verificou-se que o risco de TEV associado a varizes é alto, mas diminui com a idade: OR 4,2 aos 45 anos, 1,9 aos 60 anos e 0,9 aos 75 anos ${ }^{3}(\mathbf{B})$. Estudo de coorte não conseguiu demonstrar que a presença de varizes está independentemente associada à $\operatorname{TEV}^{11}(\mathbf{B})$. Pacientes clínicos com varizes recebendo enoxaparina $40 \mathrm{mg}$ obtiveram uma RRR de TEV de $74 \%$, a incidência de TEV foi de $21,3 \%$ vs. $5,1 \%$ nos grupos placebo e tratamento, respectivamente ${ }^{4}(\mathbf{C})$.

\section{Obesidade}

Mulheres com índice de massa corpórea acima de 27 não apresentaram correlação entre obesidade, como objetivo secundário, e TEV em análise feita post $\operatorname{hoc}^{12}(\mathbf{C})$. Estudo com 7.227 necropsias encontrou maior incidência de TEV em obesos (67\%), do que em não obesos (14\%), RR 2,97 (IC de $95 \% 1,78-4,93){ }^{13}(\mathbf{C})$. Outro trabalho encontrou um RR de 2,7 (IC de 95\% 1,3-5,8) para obesos mórbidos (IMC > 40) ${ }^{14}$ (B). Estudo caso-controle mostrou que IMC $<30$ aumentava em duas vezes a chance de trombose (IC de 95\% 1,5-3,4), quando ajustado para idade e gênero ${ }^{15}(\mathbf{B})$. Estudo de coorte encontrou associação entre obesidade (IMC > 29) e TEV em mulheres, RR 2,9 (IC de $95 \% 1,5-5,4){ }^{16}(\mathbf{A})$.

Não há evidência do benefício decorrente de estudo desenhado para avaliar a tromboprofilaxia de pacientes obesos. Em análise de subgrupos, que deve ser vista com cautela, a profilaxia em pacientes obesos, agudamente enfermos, que são internados, mostrou incidência de TEV no grupo placebo de $15,2 \%$ vs. $7,5 \%$, no grupo recebendo enoxaparina $40 \mathrm{mg} / \mathrm{dia}$. Esta diferença não atingiu significância estatística, pois 0 número de pacientes obesos foi pequeno ${ }^{4}(\mathbf{D})$.

\section{Trombofilias}

As trombofilias hereditárias, em especial deficiências de antitrombina III (ATIII), de proteína C (PC), de proteína S (PS) e resistência à proteína $C$ ativada, freqüentemente causada pela presença do fator $\mathrm{V}$ de Leiden (FVL), são distúrbios da coagulação bem estabelecidos como fatores de risco para TEV. O risco associado à trombofilia familiar, quando corrigido para o efeito de variações regionais e de gênero foi de $16,4\left(I C^{95 \%} 9,6-28,0\right)^{17}$ (B). A ATIII leva a risco maior de TEV que os outros defeitos genéticos: RR de 4,4 para ATIII vs. FVL, de 2,6 para ATIII vs. PS e de 1,9 para ATIII vs. PC ${ }^{18}(\mathbf{B})$. Portadores de deficiências de ATIII, PC ou PS, situações como cirurgia, trauma, ou imobilização, gravidez/ puerpério e uso de contracepção oral aumentam o risco de TEV: RR de 8,3, 8,2 e 6,4, respectivamente ${ }^{19}(\mathbf{B})$. Estudos do tipo casocontrole destacaram que a hiperhomocisteinemia é fator de risco não só para aterosclerose e doença vascular, mas também para TEV, com OR de 2,5 (IC de $95 \% 1,8-3,5){ }^{20}(\mathbf{B})$. 


\section{Tabela 1 - Evidência e recomendações específicas de profilaxia contra TEV em pacientes com condições clínicas diversas}

\begin{tabular}{|c|c|c|}
\hline Condição & Método & Dose \\
\hline História prévia de TEV + situações de risco & $\begin{array}{l}\text { HNF (D) } \\
\text { HBPM (D) }\end{array}$ & $\begin{array}{c}5.000 \mathrm{UI} 8-8 \mathrm{~h} \\
\text { Dalteparina (5.000 UI por dia) ou enoxaparina (40 mg por dia) }\end{array}$ \\
\hline Insuficiência venosa crônica/varizes & $\begin{array}{l}\text { Enoxaparina }^{4}(\mathbf{C}) \\
\text { Dalteparina (D) }\end{array}$ & $\begin{array}{l}40 \mathrm{mg} \text { por dia } \\
5.000 \text { UI por dia }\end{array}$ \\
\hline Obesidade + situações de risco & $\begin{array}{l}\text { Enoxaparina }{ }^{4}(\mathrm{C}) \\
\text { Dalteparina }(\mathrm{D})\end{array}$ & $\begin{array}{l}40 \mathrm{mg} \text { por dia } \\
5.000 \text { UI por dia }\end{array}$ \\
\hline
\end{tabular}

(40 mg por dia) ou warfarina (RNI 2-3)

TEV, tromboembolismo venoso; HBPM, heparina de baixo peso molecular; HNF, heparina não fracionada; RNI, razão normatizada internacional.

Figura 1 - Algoritmo para avaliação da necessidade de profilaxia de TEV em pacientes clínicos HOSPITALIZADOS (D).

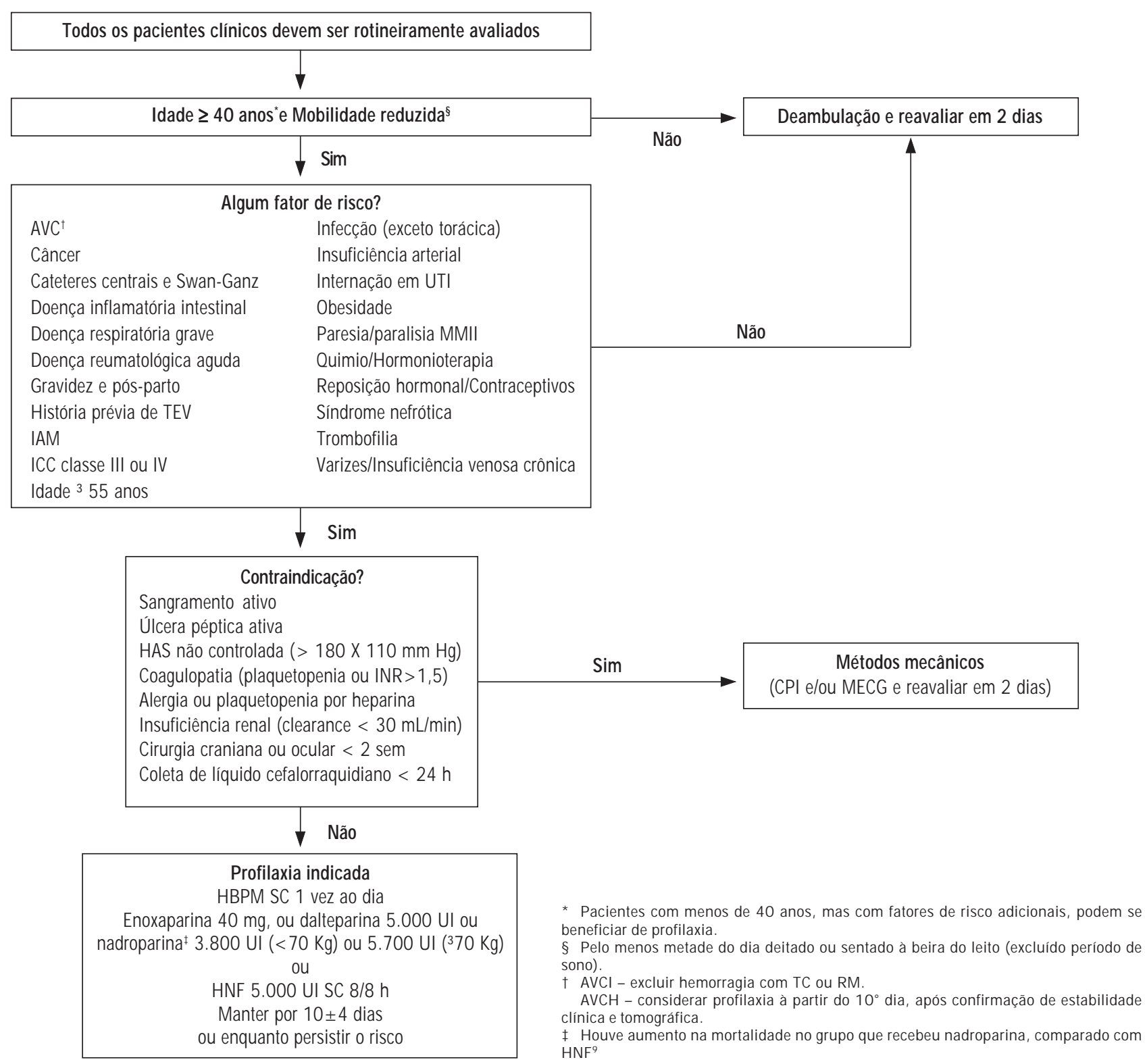


Não existem ensaios randomizados avaliando exclusivamente a eficácia da profilaxia contra TEV, em portadores de trombofilias. Poucos estudos prospectivos de coorte têm tentado avaliar a incidência de TEV em portadores de trombofilias, ocorrendo espontaneamente ou concomitantemente com outras situações de risco, como imobilização, gravidez/ puerpério e cirurgias. Notou-se que, durante períodos de risco, a incidência de TEV confirmada objetivamente foi de $4,5 \%$, naqueles recebendo profilaxia e de $16,7 \%$, naqueles sem profilaxia, RR 0,27 (IC de 95\% 0,03-2,40) ${ }^{21}$ (B). Em estudo com seguimento médio de 4 anos, mostrou-se que, durante períodos de risco, a incidência de TEV foi de $9,1 \%$, naqueles recebendo profilaxia com HNF ou HBPM, e de $21,7 \%$, naqueles sem profilaxia, RR 0,42 (IC de $95 \% 0,06-3,16)^{22}$ (B). Havendo, portanto, benefício da quimioprofilaxia somente nas situações clínicas ou cirúrgicas que aumentem o risco de TEV.

Em pacientes sintomáticos, a presença de anticoagulante lúpico está significativamente associada à TVP. Os autores detectaram a presença em 5 dos 59 pacientes $(8,5 \%)$ com TVP confirmada por venografia e em nenhum dos 117 pacientes com venografias normais $(p=0,007$ ), levando a um OR de 10,7 (IC de $95 \% 1,2-94,2)^{23}(\mathbf{B})$. Outros estudos mostrando a importância das trombofilias adquiridas como fatores de risco para TEV são discutidos em conjunto com outras condições como síndrome nefrótica, doenças reumatológicas ativas e doenças inflamatórias intestinais.

\section{Referências}

1. Motykie GD, Caprini J A, Arcelus J I, Zebala LP, Lee CE, Finke NM, et al. Risk factor assessment in the management of patients with suspected deep venous thrombosis. Int Angiol. 2000;19:47-51

2. Samama MM. An epidemiologic study of risk factors for deep vein thrombosis in medical outpatients: the Sirius study. Arch Intern Med. $2000 ; 160: 3415-20$.

3. Heit J A, Silverstein MD, M ohr DN, Petterson TM, O'Fallon WM, Melton LJ 3 rd. Risk factors for deep vein thrombosis and pulmonary embolism: a populationbased case-control study. Arch Intern Med. 2000;160:809-15.

4. Alikhan R, Cohen AT, Combe S, Samama M M, Desjardins L, Eldor A, et al. Prevention of venous thromboembolism in medical patients with enoxaparin: a subgroup analysis of the MEDENOX study. Blood Coagul Fibrinolysis. 2003;14:341-6.

5. Weill-Engerer S, Meaume S, Lahlou A, Piette F, Saint-J ean O, Sachet A, et al. Risk factors for deep vein thrombosis in inpatients aged 65 and older: a casecontrol multicenter study. J Am Geriatr Soc. 2004;52:1299-304.

6. Strekerud F, Johansen AM, Abildgaard U.Venous thromboembolismincidence and risk factors in Oslo. Tidsskr N or Laegeforen. 1998;118:3934-8. 7. Anderson FA J r, Wheeler HB, Goldberg RJ , Hosmer DW, Patwardhan NA, J ovanovic $B$, etal. A population-based perspective of the hospital incidence and case-fatality rates of deep vein thrombosis and pulmonary embolism. The Worcester DVT Study. Arch Intern Med. 1991;151:933-8.
8. Bosson J L, Labarere J, Sevestre MA, Belmin J, Beyssier L, Elias A, et al. Deep vein thrombosis in elderly patients hospitalized in subacute care facilities: a multicenter cross-sectional study of risk factors, prophylaxis, and prevalence. Arch Intern Med. 2003;163:2613-8.

9. Oger E, Leroyer C, Le Moigne E, Pomey MP, Bressollette L, Clavier J, et al. The value of a risk factor analysis in clinically suspected deep venous thrombosis. Respiration. 1997;64:326-30.

10. Ferrari E, Baudouy M, Cerboni P, Tibi T, Guigner A, Leonetti J, et al. Clinical

epidemiology of venous thromboembolic disease. Results of a French Multicentre Registry. Eur Heart J. 1997;18:685-91.

11. Goldhaber SZ, Savage DD, Garrison RJ,Castelli WP, Kannel WB, $\mathrm{McN}$ amara PM, et al. Risk factors for pulmonary embolism. The Framingham Study. Am J Med. 1983;74:1023-8.

12. G rady D, W enger N K, H errington D, Khan S, Furberg C, H unninghake $D$, et al. Postmenopausal hormone therapy increases risk for venous thromboembolic disease. The Heart and Estrogen/progestin Replacement Study. Ann Intern Med. 2000;132:689-96.

13. Blaszyk H, W ollan PC, W itkiewicz AK, Bjornsson J. D eath from pulmonary thro mbo embolism in severe o besity: lack of association with established genetic and clinical risk factors. Virchows Arch. 1999;434:529-32.

14. Tsai AW, Cushman M, Rosamond WD, Heckbert SR, Polak JF, Folsom AR Cardiovascular risk factors and venous thromboembolism incidence: the longitudinal investigation of thromboembolism etiology. Arch Intern Med.

2002;162:1182-9.

15. Abdollahi M, C ushman M, Rosendaal FR.O besity: risk of venous thrombosis and the interaction with coagulation factor levels and oral contraceptive use. Thromb Haemost. 2003;89:493-8.

16. Goldhaber SZ, Grodstein F, Stampfer MJ, Manson JE, Colditz GA, Speizer $\mathrm{FE}$, et al. A prospective study of risk factors for pulmonary embolism in women. JAMA. 1997;277:642-5.

17. Vossen CY, Conard J, Fontcuberta J, Makris M, Van Der Meer FJ, Pabinger I, et al. Familial thrombo philia and lifetime risk of venous thrombosis. J Thromb Haemost. 2004;2:1526-32.

18. Bucciarelli P, Rosendaal FR, Tripo di A, Mannucci PM, D e Stefano V, Palareti $G$,et al. Risk of venous thromboembolism and clinical manifestations in carriers of antithrombin, protein $\mathrm{C}$, protein $\mathrm{S}$ deficiency, or activated protein $\mathrm{C}$ resistance: a multicenter collaborative family study. Arterioscler Thromb Vasc Biol. 1999;19:1026-33.

19. Simioni P, Sanson BJ, Prandoni P, Tormene D, Friederich PW, G irolami B, et al. Incidence of venous thromboembolism in families with inherited thrombophilia. Thromb Haemost. 1999;81:198-202.

20. den Heijer M, Rosendaal FR, Blom HJ, Gerrits WB, Bos GM. $\mathrm{H}$ yperhomocysteinemia and venous thrombosis: a meta-analysis. Thromb H aemost. 1998;80:874-7.

21. Sanson BJ, Simioni P, Tormene D, M oia M, Friederich PW, H uisman MV, et al. The incidence of venous thromboembolism in asymptomatic carriers of a deficiency of antithrombin, protein $C$, or protein $\mathrm{S}$ : a prospective cohort study. Blood. 1999;94:3702-6.

22. Simioni P, Tormene D, Prandoni P,Zerbinati P, G avasso S, C efalo P, et al. Incidence of venous thromboembolism in asymptomatic family members who are carriers of factor $V$ Leiden: a prospective cohort study. Blood. 2002;99:1938-42.

23. Simioni P, Prandoni P, Zanon E, Saracino MA, Scudeller A, Villalta S, et al. D eep venous thrombosis and lupus anticoagulant. A case-control study. Thromb Haemost. 1996;76:187-9. 